University of Warwick institutional repository: http://go.warwick.ac.uk/wrap This paper is made available online in accordance with publisher policies. Please scroll down to view the document itself. Please refer to the repository record for this item and our policy information available from the repository home page for further information.

To see the final version of this paper please visit the publisher's website. Access to the published version may require a subscription.

Author(s): $\quad$ G. Ansaripour, G. Braithwaite, M. Myronov, O. A. Mironov, E. H. C. Parker, and T. E. Whall

Article Title: Energy loss rates of two-dimensional hole gases in inverted $\mathrm{Si} / \mathrm{Si}_{0.8} \mathrm{Ge}_{0.2}$ heterostructures

Year of publication: 2000

Link to published version: http://dx.doi.org/10.1063/1.125963

Publisher statement: None 


\title{
Energy loss rates of two-dimensional hole gases in inverted $\mathrm{Si} / \mathrm{Si}_{0.8} \mathrm{Ge}_{0.2}$ heterostructures
}

\author{
G. Ansaripour, ${ }^{\text {a) }}$ G. Braithwaite, M. Myronov, O. A. Mironov, E. H. C. Parker, \\ and T. E. Whall \\ Department of Physics, Warwick University, Coventry CV4 7AL, United Kingdom
}

(Received 13 May 1999; accepted for publication 6 January 2000)

\begin{abstract}
We have investigated the energy loss rate of hot holes as a function of carrier temperature $T_{C}$ in $p$-type inverted modulation-doped (MD) $\mathrm{Si} / \mathrm{SiGe}$ heterostructures over the carrier sheet density range $(3.5-13) \times 10^{11} \mathrm{~cm}^{-2}$, at lattice temperatures of 0.34 and $1.8 \mathrm{~K}$. It is found that the energy loss rate (ELR) depends significantly upon the carrier sheet density, $n_{2 \mathrm{D}}$. Such an $n_{2 \mathrm{D}}$ dependence of ELR has not been observed previously in $p$-type SiGe MD structures. The extracted effective mass decreases as $n_{2 \mathrm{D}}$ increases, which is in agreement with recent measurements on a gated inverted sample. It is shown that the energy relaxation of the two-dimensional hole gases is dominated by unscreened acoustic phonon scattering and a deformation potential of $3.0 \pm 0.4 \mathrm{eV}$ is deduced. (c) 2000 American Institute of Physics. [S0003-6951(00)02809-6]
\end{abstract}

A detailed quantitative understanding of hot carrier energy loss in two-dimensional (2D) carrier gas systems has yet to be realized. ${ }^{1} \mathrm{Si} / \mathrm{SiGe}$ and $\mathrm{Ge} / \mathrm{SiGe}$ structures provide new vehicles for the study of this important topic and have revealed several unusual features. For example, Song et al. ${ }^{2}$ claimed to have observed 2D phonon behavior in a strained Ge sample. In the present article, we address the question of energy relaxation by emission of 3D acoustic phonons and the controversy concerning the role of screening. Stöger et $a .^{3}$ have found that they can explain their energy loss rates (ELR) for the 2D electron gas in strained silicon by taking static screening into account. Xie et al., ${ }^{4}$ on the other hand, find evidence for weak screening in a strained $\mathrm{Si}_{0.8} \mathrm{Ge}_{0.2} 2 \mathrm{D}$ hole gas, and explain other discrepancies between theory and experiment in terms of an additional piezoelectric coupling. Braithwaite et al. ${ }^{5}$ have made further measurements on $\mathrm{Si}_{0.8} \mathrm{Ge}_{0.2}$, confirm the need for a weak screening approach and point out that static screening gives much too large a value for the deformation potential.

The present experiments have been carried out on $\mathrm{Si} / \mathrm{Si}_{0.8} \mathrm{Ge}_{0.2} / \mathrm{Si}$ heterostructures which have been modulation-doped (MD) by growing the Si:B doping slab before the alloy (inverted structure) as opposed to after alloy growth (normal structure), in contrast to previous work. ${ }^{4,5}$ Furthermore, we have investigated the carrier sheet density dependence of the ELR for the first time in an inverted SiGe heterostructure. Sample growth and preparation have been described previously. ${ }^{6(a)}$ In the inverted structure, the surface states on the silicon cap strongly affect the hole sheet density in the channel, which can be changed by reducing the silicon cap thickness using chemical etching. Assuming that the surface states pin the Fermi level in, say, midgap in $\mathrm{Si}$, a reduction in cap thickness will pull the valence band maximum down at the bottom $\mathrm{Si} / \mathrm{SiGe}$ interface, thereby reducing the carrier density.

Hall effect measurements at $0.4 \mathrm{~T}$ and $4 \mathrm{~K}$ give carrier

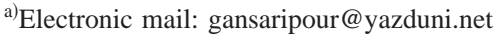

mobilities between $3200 \mathrm{~cm}^{2} \mathrm{~V}^{-1} \mathrm{~s}^{-1}$ at a carrier sheet density of $3.5 \times 10^{11} \mathrm{~cm}^{-2}$ and $1500 \mathrm{~cm}^{2} \mathrm{~V}^{-1} \mathrm{~s}^{-1}$ at 13 $\times 10^{11} \mathrm{~cm}^{-2}$. The carrier densities agree with those obtained from measurements of Shubnikov-de Haas $(\mathrm{SdH})$ oscillations in the resistance $R$ as the magnetic field $B$ is increased. For example, from the Hall effect, we obtain $5.3 \pm 0.3$ $\times 10^{11} \mathrm{~cm}^{-2}$, while the resistivity obtained from the $\mathrm{SdH}$ period in $\mathrm{B}^{-1}$ is $5.6 \pm 0.4 \times 10^{11} \mathrm{~cm}^{-2}$. The results are consistent with the absence of parallel conduction, i.e., conduction at a single heterointerface in a single 2D subband. PoissonSchrödinger calculations have confirmed that the position of the Fermi level is in the lowest subband. The SdH oscillations were examined in fields $B<4 \mathrm{~T}$ for which there is only one peak in the fast Fourier transform, Fig. 1, corresponding to one frequency of oscillation, and for which the condition $\Delta R / R<0.1$ applies. ${ }^{7}$ Electric field $(F)$ heating of carriers, at a lattice temperature $T_{L}$ of $0.34 \mathrm{~K}$, was investigated by $\mathrm{SdH}$ thermometry. ${ }^{5}$ The temperature dependence of the amplitude of the $\mathrm{SdH}$ oscillations was measured for infinitesimal electric fields $\left(<0.2 \mathrm{~V} \mathrm{~m}^{-1}\right)$ and then, holding the lattice temperature at $0.34 \mathrm{~K}$, different currents were passed through the sample to provide carrier heating in the electric field $(F)$ range of $0.2-140 \mathrm{~V} \mathrm{~m}^{-1}$. The carrier temperature was deduced by measuring the amplitude of the $\mathrm{SdH}$ oscillation,

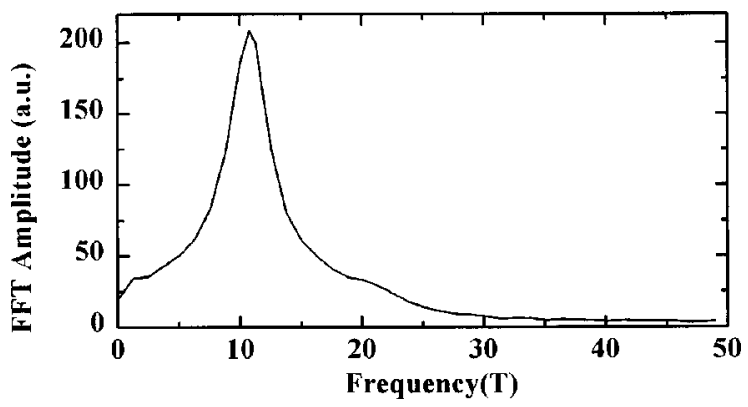

FIG. 1. Typical fast Fourier transform of the Shubnikov-de Haas oscillations observed in the present inverted MD samples, taken up to a field of 5 $\mathrm{T}$. For fields below $4 \mathrm{~T}$, the shoulder at a frequency of $22 \mathrm{~T}$ (and associated with spin splitting) is absent. 


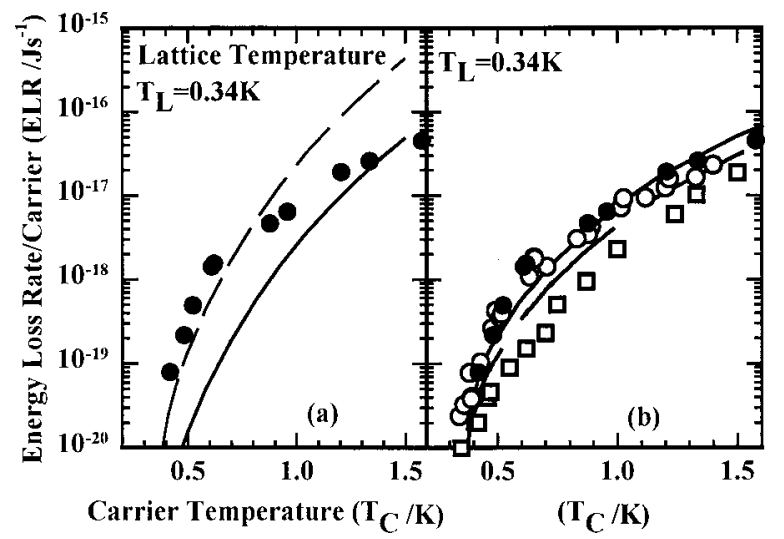

FIG. 2. (a) Experimental energy loss rate per carrier vs carrier temperature for an inverted MD sample with sheet density $5.1 \times 10^{11} \mathrm{~cm}^{-2}$ and Ge content $x=0.2$ (symbols). Lines represent theoretical calculations for fully screened deformation potential scattering with $D_{\mathrm{ac}}=20 \mathrm{eV}$ (solid) and $D_{\mathrm{ac}}$ $=60 \mathrm{eV}$ (dashed). Effective mass used $m^{*}=0.29 m_{0}$ and (b) ELR vs $T_{C}$ for the same inverted sample as in (a) and two normal MD SiGe samples along with theoretical calculations for an unscreened potential. Inverted 5.1 $\times 10^{11} \mathrm{~cm}^{-2}$ ( Experiment), normal $5.6 \times 10^{11} \mathrm{~cm}^{-2}(\square$ Experiment, dashed line-theory, $\left.m^{*}=0.3 m_{0}, D_{\mathrm{ac}}=3 \mathrm{eV}\right)$, normal $4.8 \times 10^{11} \mathrm{~cm}^{-2}(\bigcirc$ Experiment, solid line-theory, $\left.m^{*}=0.3 m_{0}, D_{\mathrm{ac}}=3 \mathrm{eV}\right)$.

and by comparison with the lattice temperature dependence of the $\mathrm{SdH}$ amplitude. Since the $\mathrm{SdH}$ oscillation was measured at constant current (i.e., varying $I^{2} R$ product), an interpolation method was used to determine the carrier temperature under constant power.

Although typical carrier mobilities were $\sim 3600$ $\mathrm{cm}^{2} \mathrm{~V}^{-1} \mathrm{~s}^{-1}$, at a lattice temperature $\geqslant 1.8 \mathrm{~K}$, it was found that the $\mathrm{SdH}$ oscillations were not as well pronounced when compared to lower temperatures. Therefore, to examine electric field heating at this higher lattice temperature, the analogous method of zero magnetic field conductance thermometry was used, as in our previous work. ${ }^{5}$ Hall effect measurements were used to deduce the carrier mobility $\mu$ at a carrier temperature $T_{C}$ and the ELR per carrier, equal to $e \mu F^{2}$, was then determined.

The mean ELR due to inelastic scattering by acoustic phonons is given by ${ }^{8}$

$$
\left\langle\frac{d E}{d t}\right\rangle=\frac{\int_{0}^{\infty} \int_{0}^{2 \pi} f\left(E, T_{C}\right) \hbar \omega_{q}\left[\nu_{+}(\theta)-\nu_{-}(\theta)\right] d \theta d E}{\int_{0}^{\infty} E f\left(E, T_{C}\right) d E}
$$

with

$$
\begin{aligned}
\nu_{ \pm}(\theta)= & \int_{-\infty}^{\infty} d q_{z}\left|I\left(q_{z}\right)\right|^{2} P\left(q_{x, y}, q_{z}\right)\left(N_{q}+\frac{1}{2} \pm \frac{1}{2}\right) \\
& \times\left[1-f\left(E \pm \hbar \omega_{q}, T_{C}\right)\right] \Theta\left(E-\frac{\hbar \omega_{q}}{2} \pm \frac{\hbar \omega_{q}}{2}\right),
\end{aligned}
$$

where $\theta$ is the scattering angle, $\nu_{+}(\theta)$, refers to phonon emission, $\nu_{-}(\theta)$ to phonon absorption, $q_{z}$ and $q_{x y}$ are the wave vector components perpendicular and parallel to the heterointerface, $N_{q}$ is the phonon occupation number at wave vector $q=\left(q_{x y}^{2}+q_{z}^{2}\right)^{1 / 2}$ and lattice temperature $T_{L}, \Theta$ is the Heaviside function, $f\left(E, T_{C}\right)$ the Fermi Dirac distribution for a carrier temperature $T_{C}, I\left(q_{z}\right)$ is the form factor for the Fang-Howard wave function, and $\hbar \omega_{q}$ is the acoustic phonon energy. The acoustic phonon scattering probability is

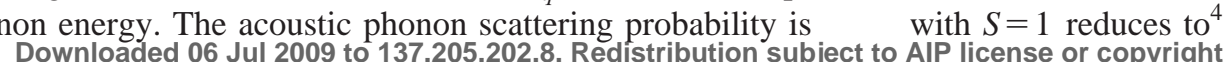

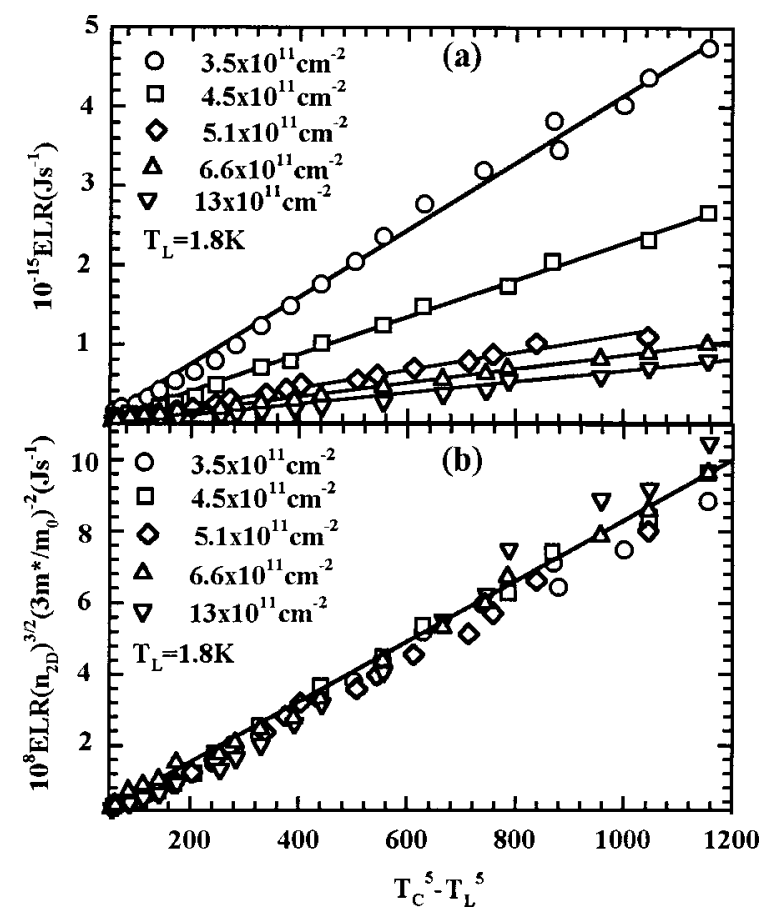

FIG. 3. (a) Measured energy loss rate per carrier (ELR) for samples of carrier sheet densities $(3.5-13) \times 10^{11} \mathrm{~cm}^{-2}$, plotted vs $T_{C}^{5}-T_{L}^{5}$, and (b) normalized energy loss rate plotted vs $T_{C}^{5}-T_{L}^{5}$.

$$
P\left(q_{x y}, q_{z}\right)=\frac{m^{*} D_{\mathrm{ac}}^{2} u_{L}(x) q}{8 \pi^{2} \hbar c_{L}(x) S^{2}\left(E, T_{C}, q\right)},
$$

where $S\left(E, T_{C}, q\right)$ is the static screening factor, ${ }^{8} c_{L}(x)$ is the longitudinal elastic coefficient, $m^{*}$ is the in-plane effectivemass, $u_{L}$ is the longitudinal sound velocity, and $D_{\text {ac }}$ is the acoustic phonon deformation potential. The theoretically predicted maximum values of $S^{2}\left(E, T_{C}, q\right)$ are between 6.5 (for $n_{2 \mathrm{D}}=13 \times 10^{11} \mathrm{~cm}^{-2}$ ) and 16 (for $3.5 \times 10^{11} \mathrm{~cm}^{-2}$ ).

Figure 2(a) shows the measured energy loss rate for an inverted structure of sheet density $5.1 \times 10^{11} \mathrm{~cm}^{-2}$, at a constant lattice temperature of $0.34 \mathrm{~K}$. Also shown is an attempt to fit our experimental data using a screened scattering potential with $S>1$. Depending on the value of $D_{\text {ac }}$ chosen, we only obtain a fit at low $T_{C}\left(D_{\mathrm{ac}}=60 \mathrm{eV}\right)$ or at high $T_{C}\left(D_{\mathrm{ac}}\right.$ $=20 \mathrm{eV}$ ) but are unable to obtain a fit over the whole carrier temperature range. Moreover, the values of $D_{\mathrm{ac}}$ are considerably in excess of what is reported for bulk $\mathrm{Si}$ and $\mathrm{Ge}$ (i.e., 4 and $3.5 \mathrm{eV}$, respectively), ${ }^{9}$ and are therefore regarded as unrealistic.

We next attempt to fit the data by assuming $S=1$ (weak screening), as shown in Fig. 2(b). Experiment and theory now agree within experimental error, for both the normal and inverted structures, with a very plausible value of $D_{\text {ac }}$ $=3 \mathrm{eV}$. In order to further test the validity of the weak screening assumption, we now consider the experimental data for four inverted MD SiGe samples of various carrier densities, at a lattice temperature of $1.8 \mathrm{~K}$. At low enough temperatures where the phonon population is small (BlochGruneisen regime), the carrier gas is degenerate and the Pauli exclusion restricts the allowed scattering processes, Eq. (1)

作 


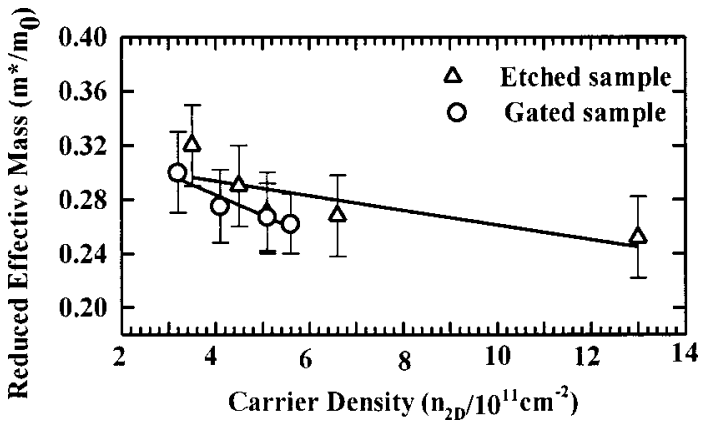

FIG. 4. Comparison between the extracted effective masses of the etched samples and those measured on a gated sample [see Ref. 6(b)].

$$
\mathrm{ELR}=\frac{m^{* 2} D_{\mathrm{ac}}^{2} k_{B}^{5}(4 !) \zeta(5)}{8 \sqrt{2} \pi^{5 / 2}\left(n_{2 \mathrm{D}}\right)^{3 / 2} \rho u_{L}^{4} \hbar^{7}}\left(T_{C}^{5}-T_{L}^{5}\right),
$$

where $\rho$ is the mass density and $n_{2 \mathrm{D}}$ is carrier sheet density. In Fig. 3(a), the experimental ELR are plotted against $T_{C}^{5}$ $-T_{L}^{5}$ and a linear dependence is demonstrated. It is also seen that the power loss of the investigated samples depends upon the carrier sheet density. This carrier sheet density dependence of the energy loss rate has not previously been observed in similar normal MD Si/SiGe samples. ${ }^{5}$ This is also in contrast to the results given by Stöger $e t ~ a l .{ }^{3}$ who found no dependence of ELR on carrier density for the 2D electron gas in strained silicon. We note from Eq. (4) that the ELR should vary as $\left(T_{C}^{5}-T_{L}^{5}\right) m^{* 2}\left(n_{2 \mathrm{D}}\right)^{-3 / 2}$. The data are therefore replotted in Fig. 3(b) as normalized energy loss rate $\left[\operatorname{ELR}\left(n_{2 \mathrm{D}}\right)^{3 / 2} m^{*-2}\right]$ vs $T_{C}^{5}-T_{L}^{5}$ and by adjusting $m^{*}$ to obtain a universal straight line. The $m^{*}$ so obtained are compared in Fig. 4 with effective mass measurements on a gated inverted structure $^{6(b)}$ and it is seen that they agree to within experimental error. Having demonstrated that the weak screening approach gives a satisfactory description of the variation of ELR with effective mass and carrier density, we compute the average value of $D_{\mathrm{ac}}$ for the complete set of data from the exact theory for an unscreened potential, Eq. (1) with $S=1$, and as before obtain $3.0 \pm 0.4 \mathrm{eV}$.

To summarize, the static screening approach does not seem to work for the present samples, since we need to assume unreasonably small values of the static screening factor $S$ in order to fit our results. A possible explanation is provided by Fischetti and Laux ${ }^{10}$ who point out that dynamic screening of the acoustic phonons should apply at low enough temperatures and/or high carrier densities. They also point out that the theoretical treatment is quite intractable at present, but it is reasonable to expect that such screening might be quite weak. Challis et al. ${ }^{11}$ also question the procedures used to account for screening of a deformation potential and note that the screening might, in any event, be reduced by other scattering mechanisms. In the case of the $\mathrm{Si} / \mathrm{SiGe}$ system, the question as to why static screening seems to be appropriate for electrons ${ }^{3}$ but not for holes remains to be answered.

In conclusion, we have investigated the energy loss rate of holes in $p$-type inverted modulation-doped Si/SiGe heterostructures. The results support an interpretation in terms of weak screening of the acoustic phonon deformation potential, in contrast to the behavior seen in the $\mathrm{Si} / \mathrm{SiGe}$ electron gas.

The authors are grateful to Professors G. Bauer and L. Smrcka for valuable discussions on the general topic of carrier heating. They thank P. J. Phillips, C. P. Parry for layer growth, and M. A. Sadeghzadeh for sample processing. One of the authors (G.A.) wishes to acknowledge the award of a $\mathrm{Ph} . \mathrm{D}$. scholarship by the Government of Iran.

${ }^{1}$ B. K. Ridley, Rep. Prog. Phys. 54, 169 (1991).

${ }^{2}$ S. H. Song, Wei Pan, D. C. Tsui, Y. H. Xie, and D. Monroe, Appl. Phys. Lett. 70, 3422 (1999).

${ }^{3}$ G. Stöger, G. Brunthaler, G. Bauer, K. Ismail, B. S. Meyerson, J. Lutz, and F. Kuchar, Phys. Rev. B 49, 10417 (1993).

${ }^{4}$ Y. H. Xie, R. People, J. C. Bean, and W. Wecht, Appl. Phys. Lett. 49, 283 (1986).

${ }^{5}$ G. Braithwaite, N. L. Mattey, E. H. C. Parker, T. E. Whall, G. Brunthaler, and G. Bauer, J. Appl. Phys. 81, 6853 (1997). The calculation of the deformation potential in this paper contains an arithmetic error in the effective mass and is therefore incorrect. A recalculation gives $D_{\text {ac }}=3.0$ $\pm 0.5 \mathrm{eV}$ which agrees with that obtained in the present work.

${ }^{6}$ (a)M. A. Sadeghzadeh, C. P. Parry, P. J. Phillips, E. H. C. Parker, and T. E. Whall, Appl. Phys. Lett. 74, 579 (1999); ${ }^{6}(\mathrm{~b})$ Proceedings of XXVII Intern. School on Physics of Semiconducting Compounds, Jazowies (1998).

${ }^{7}$ P. T. Coleridge, R. Stoner, and R. Fletcher, Phys. Rev. B 39, 1120 (1998).

${ }^{8}$ K. Hirakawa and H. Sakaki, Appl. Phys. Lett. 49, 889 (1986).

${ }^{9}$ J. D. Wiley, in Semiconductors and Semimetals, edited by R. K. Willardson and A. C. Beer (Academic, New York, 1975), Vol. 10, p. 91.

${ }^{10}$ M. V. Fischetti and S. E. Laux, Phys. Rev. B 48, 2244 (1993).

${ }^{11}$ L. J. Challis, G. A. Toombs, and F. W. Sheard, Lectures at the Karpacz Winter School on Phonon Physics, February 1987. 